\title{
Damping-Antidamping Effect on Comets Motion
}

\author{
G. V. López, E. M. Juárez \\ Departamento de Fsica, Universidad de Guadalajara, Guadalajara, México \\ Email: gulopez@cencar.udg.mx
}

Received September 12, 2013; revised October 15, 2013; accepted November 11, 2013

Copyright (C) 2013 G. V. López, E. M. Juárez. This is an open access article distributed under the Creative Commons Attribution License, which permits unrestricted use, distribution, and reproduction in any medium, provided the original work is properly cited.

\begin{abstract}
We make an observation about Galilean transformation on a 1-D mass variable system which leads us to the right way to deal with mass variable systems. Then using this observation, we study two-body gravitational problem where the mass of one of the bodies varies and suffers a damping-antidamping effect due to star wind during its motion. For this system, a constant of motion, a Lagrangian and a Hamiltonian are given for the radial motion, and the period of the body is studied using the constant of motion of the system. Our theoretical results are applied to Halley's Comet.
\end{abstract}

Keywords: Quantum Computer; Controlled-Not Gate; Diamond

\section{Introduction}

There is no doubt that mass variable systems have been relevant since the foundation of the classical mechanics and modern physics [1]. These types of systems have been known as Gylden-Meshcherskii problems [2-9], and among these types of systems one could mention: the motion of rockets [10], the kinetic theory of dusty plasma [11], propagation of electromagnetic waves in a dispersive nonlinear media [12], neutrinos mass oscillations [13] and [14], black holes formation [15], and comets interacting with solar wind [16]. This last system belongs to the so called "gravitational two-body problem" which is one of the most studied and well known system in classical mechanics [17]. In this type of system, one assumes normally that the masses of the bodies are fixed and unchanged during the dynamical motion. However, when one is dealing with comets, besides considering its mass variation due to the interaction with the solar wind, one would like to have an estimation of the the effect of the solar wind pressure on the comet motion. This pressure may produce a dissipative-antidissipative effect on its motion. The dissipation effect must be felt by the comet when this one is approaching to the sun (or star), and the antidissipation effect must be felt by the comet when this one is moving away from the sun. To deal with these types of mass variation problem, it has been proposed that the Newton equation must be modified [10] and [18] since the system becomes noninvariant under change of inertial systems (Galileo transformation).
In this paper, we will make a first observation about this statement which indicates that such a proposed modification of Newton's equation has some problems and rather the use of the original Newton equation is the right approach to dealing with mass variation systems, which was used in previous paper [19] to study two-bodies gravitational problem with mass variation in one of them, where we were interested in the difference of the trajectories in the spaces $(x, v)$ and $(x, p)$. As a consequence, there is an indication that mass variation problems must be dealt with as noninvariant under Galilean transformation. Second, we study the two-body gravitational problem taking into consideration the mass variation of one of them and its damping-antidamping effect due to the solar wind. The mass of the other body is assumed big and fixed, and the reference system of motion is chosen just in this body. In addition, we will assume that the mass lost is expelled from the body radially to its motion. Doing this, the three-dimensional two-body problem is reduced to a one-dimensional problem. Then, a constant of motion, the Lagrangian, and the Hamiltonian are deduced for this one-dimensional problem, where a radial dissipative antidissipative force proportional to the velocity square is chosen. A model for the mass variation is given, and the dampingantidamping effect is studied on the period of the trajectories, the trajectories themselves, and the aphelion distance of a comet. We use the parameters associated to comet Halley to illustrate the application of our results. 


\section{Mass Variation Problem and Galileo Transformation}

To simplify our discussion and without losing generality, we will restrict myself to one degree of freedom. Newton equation of motion is given by

$$
\frac{\mathrm{d}}{\mathrm{d} t}(m(t) v)=F(x, v, t),
$$

where $m(t) v$ is the quantity of movement, $F$ is the total external force acting on the object, $m(t)$ and $v=\mathrm{d} x / \mathrm{d} t$ are its time depending mass and velocity of the body (motion of the mass lost is not considered). Galileo transformations to another inertial frame $\left(S^{\prime}\right)$ which is moving with a constant velocity $u$ respect our original frame $S$ are defined as

$$
\begin{gathered}
x^{\prime}=x-u t \\
t^{\prime}=t
\end{gathered}
$$

which implies the following relation between the velocity seen in the reference system $S, v$, and the velocity seen in the reference system $S^{\prime}, v^{\prime}$,

$$
v^{\prime}=v-u \text {. }
$$

Multiplying the last term by $m\left(t^{\prime}\right)$ and making the differentiation with respect to $t^{\prime}$, one gets

$$
\frac{\mathrm{d}}{\mathrm{d} t^{\prime}}\left(m\left(t^{\prime}\right) v^{\prime}\right)=F^{\prime}\left(x^{\prime}, v^{\prime}, t^{\prime}\right),
$$

where $F^{\prime}$ is given by

$$
F^{\prime}\left(x^{\prime}, v^{\prime}, t^{\prime}\right)=F\left(x^{\prime}+u t^{\prime}, v^{\prime}+u, t^{\prime}\right)-u \frac{\mathrm{d} m\left(t^{\prime}\right)}{\mathrm{d} t^{\prime}} .
$$

Therefore, Equations (1) and (5) have the same form but the force is different since in addition to the transformed force term $F\left(x^{\prime}+u t^{\prime}, v^{\prime}+u, t^{\prime}\right)$, one has the term $u \mathrm{dm}\left(t^{\prime}\right) / \mathrm{d} t^{\prime}$. This noninvariant form of the force under Galilean transformation has lead to propose [10] and [18] that Newton Equation (1) to modify Newton's equation of motion for mass variation objects, to keep the principle of invariance of equation under Galilean transformations, of the form

$$
m(t) \frac{\mathrm{d} v}{\mathrm{~d} t}=F(x, v, t)+w \frac{\mathrm{d} m(t)}{\mathrm{d} t},
$$

where $w$ is the relative velocity of the escaping mass with respect the center of mass of the object. When one does a Galilean transformation on this equation, one gets

$$
m\left(t^{\prime}\right) \frac{\mathrm{d} v^{\prime}}{\mathrm{d} t^{\prime}}=F^{\prime}\left(x^{\prime}, v^{\prime}, t^{\prime}\right)
$$

where $F^{\prime}$ is given by

$$
F^{\prime}\left(x^{\prime}, v^{\prime}, t^{\prime}\right)=F\left(x^{\prime}+u t^{\prime}, v^{\prime}+u, t^{\prime}\right)+w \frac{\mathrm{d} m\left(t^{\prime}\right)}{\mathrm{d} t^{\prime}},
$$

which has the same form as Equation (7). However, assume for the moment that $w=$ constant and $F=0$. So, from Equation (7), it follows that

$$
v(t)=v_{0}+\ln \left(\frac{m(t)}{m_{0}}\right)^{w}
$$

where $m_{0}=m(0)$. In this way, if we have a mass variation of the for $m(t)=m_{0} \mathrm{e}^{-\alpha t}$ (for example), one would have a velocity behavior like

$$
v(t)=v_{0}-w \alpha t
$$

which is not acceptable since one can have $v>0$, $v=0$ and $v<0$ depending on the value wat. Even more, since for $F=0$, the equation resulting in the reference system $S^{\prime}$ is the same, i.e. in $S^{\prime}$ one gets the same type of solution,

$$
v^{\prime}\left(t^{\prime}\right)=v_{0}+\ln \left(\frac{m\left(t^{\prime}\right)}{m_{0}}\right)
$$

which is independent on the relative motion of the reference frames, and this must not be possible due to relation (4).

In addition, it worths to mention that special theory of relativity can be seen as the motion of mass variation problem, where the mass depends on the velocity of the particle of the form $m(v)=m_{0}\left(1-v^{2} / c 2\right)^{-1 / 2}$, with $c$ being the speed of light. This system is obviously not invariant under Galilean transformation, and given the force, Newton's equation motion is always kept in the same form to solve a relativistic problem, $\mathrm{d}(m(v) v) / \mathrm{d} t=F(x, v, t),[20]$ and [1].

\section{Mass Variation and Equations of Motion}

Having explained and clarify the problem of mass variation [21], Newton's equations of motion for two bodies interacting gravitationally, seen from arbitrary inertial reference system, and with radial dissipativeantidissipative force acting in one of them are given by

$$
\frac{\mathrm{d}}{\mathrm{d} t}\left(m_{1} \frac{\mathrm{d} \boldsymbol{r}_{1}}{\mathrm{~d} t}\right)=-\frac{G m_{1} m_{2}}{\left|\boldsymbol{r}_{1}-\boldsymbol{r}_{2}\right|^{3}}\left(\boldsymbol{r}_{1}-\boldsymbol{r}_{2}\right)
$$

and

$$
\frac{\mathrm{d}}{\mathrm{d} t}\left(m_{2} \frac{\mathrm{d} r_{2}}{\mathrm{~d} t}\right)=-\frac{G m_{1} m_{2}}{\left|\boldsymbol{r}_{2}-\boldsymbol{r}_{1}\right|^{3}}\left(\boldsymbol{r}_{2}-\boldsymbol{r}_{1}\right)-\frac{\gamma}{\left|\boldsymbol{r}_{1}-\boldsymbol{r}_{2}\right|}\left[\frac{\mathrm{d}\left|\boldsymbol{r}_{1}-\boldsymbol{r}_{2}\right|}{\mathrm{d} t}\right]^{2}\left(\boldsymbol{r}_{2}-\boldsymbol{r}_{1}\right),
$$


where $m_{1}$ and $m_{2}$ are the masses of the two bodies, $\boldsymbol{r}_{1}=\left(x_{1}, y_{1}, z_{1}\right)$ and $\boldsymbol{r}_{2}=\left(x_{2}, y_{2}, z_{2}\right)$ are their vectors positions from the reference system, $G$ is the gravitational constant $\left(G=6.67 \times 10^{-11} \mathrm{~m}^{3} / \mathrm{Kg} \mathrm{s}^{2}\right), \gamma$ is the nonnegative constant parameter of the dissipativeantidissipative force, and

$\left|\boldsymbol{r}_{1}-\boldsymbol{r}_{2}\right|=\left|\boldsymbol{r}_{2}-\boldsymbol{r}_{1}\right|=\sqrt{\left(x_{2}-x_{1}\right)^{2}+\left(y_{2}-y_{1}\right)^{2}+\left(z_{2}-z_{1}\right)^{2}}$ is the Euclidean distance between the two bodies. Note that if $\gamma>0$ and $\mathrm{d}\left|\boldsymbol{r}_{1}-\boldsymbol{r}_{2}\right| / \mathrm{d} t>0$ one has dissipation since the force acts against the motion of the body, and for $\mathrm{d}\left|\boldsymbol{r}_{1}-\boldsymbol{r}_{2}\right| / \mathrm{d} t<0$ one has anti-dissipation since the force pushes the body. If $\gamma<0$ this scheme is reversed and corresponds to our actual situation with the comet mass lost.

It will be assumed the mass $m_{1}$ of the first body is constant and that the mass $m_{2}$ of the second body varies. Now, It is clear that the usual relative, $\boldsymbol{r}$, and center of mass, $R$, coordinates defined as $\boldsymbol{r}=\boldsymbol{r}_{2}-\boldsymbol{r}_{1}$ and $R=\left(m_{1} \boldsymbol{r}_{1}+m_{2} \boldsymbol{r}_{2}\right) /\left(m_{1}+m_{2}\right)$ are not so good to describe the dynamics of this system. However, one can consider the case for $m_{1} \gg m_{2}$ (which is the case star-comet), and consider to put our reference system just on the first body $\left(\boldsymbol{r}_{1}=\mathbf{0}\right)$. In this case, Equation (13) and Equation (14) are reduced to the equation

$$
m_{2} \frac{\mathrm{d}^{2} \boldsymbol{r}}{\mathrm{d} t^{2}}=-\frac{G m_{1} m_{2}}{r^{3}} \boldsymbol{r}-\dot{m}_{2} \dot{\boldsymbol{r}}-\gamma\left[\frac{\mathrm{d} r}{\mathrm{~d} t}\right]^{2} \hat{\boldsymbol{r}},
$$

where one has made the definition $\boldsymbol{r}=\boldsymbol{r}_{2}=(x, y, z), r$ is its magnitude, $r=\sqrt{x^{2}+y^{2}+z^{2}}$ and $\hat{\boldsymbol{r}}=\boldsymbol{r} / r$ is the unitary radial vector. Using spherical coordinates $(r, \theta, \varphi)$,

$$
x=r \sin \theta \cos \varphi, y=r \sin \theta \sin \varphi, z=r \cos \theta,
$$

one obtains the following coupled equations

$$
\begin{array}{r}
m_{2}\left(\ddot{r}-r \dot{\theta}^{2}-r \dot{\varphi}^{2} \sin ^{2} \theta\right)=-\frac{G m_{1} m_{2}}{r^{2}}-\dot{m}_{2} \dot{r}-\gamma \dot{r}^{2} \\
m_{2}\left(2 \dot{r} \dot{\theta}+r \ddot{\theta}-r \dot{\varphi}^{2} \sin \theta \cos \theta\right)=-\dot{m}_{2} r \dot{\theta},
\end{array}
$$

and

$$
m_{2}(2 \dot{r} \dot{\varphi} \sin \theta+r \ddot{\varphi} \sin \theta+2 r \dot{\varphi} \dot{\theta} \cos \theta)=-\dot{m}_{2} r \dot{\varphi} \sin \theta
$$

Taking $\dot{\varphi}=0$ as solution of this last equation, the resulting equations are

$$
m_{2}\left(\ddot{r}-r \dot{\theta}^{2}\right)=-\frac{G m_{1} m_{2}}{r^{2}}-\dot{m}_{2} \dot{r}-\gamma \dot{r}^{2},
$$

and

$$
m_{2}(2 \dot{r} \dot{\theta}+r \ddot{\theta})+\dot{m}_{2} r \dot{\theta}=0 .
$$

From this last expression, one gets the following constant of motion (usual angular momentum of the system)

$$
l_{\theta}=m_{2} r^{2} \dot{\theta}
$$

and with this constant of motion substituted in Equation (20), one obtains the following one-dimensional equation of motion for the radial part

$$
\frac{\mathrm{d}^{2} r}{\mathrm{~d} t^{2}}=-\frac{G m_{1}}{r^{2}}-\frac{\dot{m}_{2}}{m_{2}}\left(\frac{\mathrm{d} r}{\mathrm{~d} t}\right)-\frac{\gamma}{m_{2}} \dot{r}^{2}+\frac{l_{\theta}^{2}}{m_{2}^{2} r^{3}} .
$$

Now, let us assume that $m_{2}$ is a function of the distance between the first and the second body, $m_{2}=m_{2}(r)$. Therefore, it follows that

$$
\dot{m}_{2}=m_{2}^{\prime} \dot{r},
$$

where $m_{2}^{\prime}$ is defined as $m_{2}^{\prime}=\mathrm{d} m_{2} / \mathrm{d} r$. Thus, Equation (23) is written as

$$
\frac{\mathrm{d}^{2} r}{\mathrm{~d} t^{2}}=-\frac{G m_{1}}{r^{2}}+\frac{l_{\theta}^{2}}{m_{2}^{2} r^{3}}-\frac{m_{2}^{\prime}+\gamma}{m_{2}}\left(\frac{\mathrm{d} r}{\mathrm{~d} t}\right)^{2},
$$

which, in turns, can be written as the following autonomous dynamical system

$$
\frac{\mathrm{d} r}{\mathrm{~d} t}=v ; \frac{\mathrm{d} v}{\mathrm{~d} t}=-\frac{G m_{1}}{r^{2}}+\frac{l_{\theta}^{2}}{m_{2}^{2} r^{3}}-\frac{m_{2}^{\prime}+\gamma}{m_{2}} v^{2} .
$$

Note from this equation that $m_{2}^{\prime}$ is always a nonpositive function of $r$ since it represents the mass lost rate. On the other hand, $\gamma$ is a negative parameter in our case.

\section{Constant of Motion, Lagrangian and Hamiltonian}

A constant of motion for the dynamical system (26) is a function $K=K(r, v)$ which satisfies the partial differential equation [22]

$$
v \frac{\partial K}{\partial r}+\left[\frac{-G m_{1}}{r^{2}}+\frac{l_{\theta}^{2}}{m_{2}^{2} r^{3}} \frac{-m_{2}^{\prime}+\gamma}{m_{2}} v^{2}\right] \frac{\partial K}{\partial v}=0 .
$$

The general solution of this equation is given by [23]

$$
K(x, v)=F(c(r, v)),
$$

where $F$ is an arbitrary function of the characteristic curve $c(r, v)$ which has the following expression

$$
\begin{aligned}
c(r, v) & =m_{2}^{2}(r) \mathrm{e}^{2 \gamma \lambda(r)} v^{2} \\
& +\int\left(\frac{2 G m_{1}}{r^{2}}-\frac{2 l_{\theta}^{2}}{m_{2}^{2} r^{3}}\right) m_{2}^{2}(r) \mathrm{e}^{2 \gamma \lambda(r)} \mathrm{d} r,
\end{aligned}
$$

and the function $\lambda(r)$ has been defined as

$$
\lambda(r)=\int \frac{\mathrm{d} r}{m_{2}(r)} .
$$

During a cycle of oscillation, the function $m_{2}(r)$ can be different for the comet approaching the sun and for the comet moving away from the sun. Let us denote $m_{2+}(r)$ for the first case and $m_{2-}(r)$ for the second case. Therefore, one has two cases to consider in Equa- 
tion (28) which will denoted by $( \pm)$. Now, if $m_{2 \pm}^{o}$ denotes the mass at aphelium $(+)$ or perielium $(-)$ of the comet, $F(c)=c^{ \pm} / 2 m_{2+}^{o}$ represents the functionality in Equation (28) such that for $m_{2}$ constant and $\gamma$ equal zero, this constant of motion is the usual gravitational energy. Thus, the constant of motion can be chosen as $K^{ \pm}=c(r, v) / 2 m_{2 \pm}^{o}$, that is,

$$
K^{ \pm}=\frac{m_{2 \pm}^{2}(r)}{2 m_{2 \pm}^{o}} \mathrm{e}^{2 \gamma \lambda_{ \pm}(r)} v^{2}+V_{e f f}^{ \pm}(r),
$$

where the effective potential $V_{\text {eff }}$ has been defined as

$$
V_{\text {eff }}^{ \pm}(r)=\frac{G m_{1}}{m_{2 \pm}^{o}} \int \frac{m_{2 \pm}^{2}(r) \mathrm{e}^{2 \gamma \lambda_{ \pm}(r)} \mathrm{d} r}{r^{2}}-\frac{l_{\theta}^{2}}{m_{2 \pm}^{o}} \int \frac{\mathrm{e}^{2 \gamma \lambda_{ \pm}(r)} \mathrm{d} r}{r^{3}}
$$

This effective potential has an extreme at the point $r_{*}$ defined by the relation

$$
r_{*} m_{2}^{2}\left(r_{*}\right)=\frac{l_{\theta}^{2}}{G m_{1}}
$$

which is independent on the parameter $\gamma$ and depends on the behavior of $m_{2}(r)$. This extreme point is a minimum of the effective potential since one has

$$
\left(\frac{\mathrm{d}^{2} V_{e f f}^{ \pm}}{\mathrm{d} r^{2}}\right)_{r=r_{*}}>0
$$

Using the known expression [24-26] for the Lagrangian in terms of the constant of motion,

$$
L(r, v)=v \int \frac{K(r, v) \mathrm{d} v}{v^{2}},
$$

the Lagrangian, generalized linear momentum and the Hamiltonian are given by

$$
\begin{gathered}
L^{ \pm}=\frac{m_{2 \pm}^{2}(r)}{2 m_{2 \pm}^{o}} \mathrm{e}^{2 \gamma \lambda_{ \pm}(r)} v^{2}-V_{\text {eff }}^{ \pm}(r), \\
p=\frac{m_{2 \pm}^{2}(r) v}{m_{2 \pm}^{o}} \mathrm{e}^{2 \gamma \lambda_{ \pm}(r)},
\end{gathered}
$$

and

$$
H^{ \pm}=\frac{m_{2 \pm}^{o} p^{2}}{2 m_{2 \pm}^{2}(r)} \mathrm{e}^{-2 \gamma \lambda_{ \pm}(r)}+V_{e f f}^{ \pm}(r) .
$$

The trajectories in the space $(x, v)$ are determined by the constant of motion (31). Given the initial condition $\left(r_{o}, v_{o}\right)$, the constant of motion has the specific value

$$
K_{o}^{ \pm}=\frac{m_{2 \pm}^{2}\left(r_{o}\right)}{2 m_{2 \pm}^{o}} \mathrm{e}^{2 \gamma \lambda_{ \pm}\left(r_{o}\right)} v_{o}^{2}+V_{e f f}^{ \pm}\left(r_{o}\right),
$$

and the trajectory in the space $(r, v)$ is given by

$$
v= \pm \sqrt{\frac{2 m_{2 \pm}^{o}}{m_{2 \pm}^{2}(r)}} \mathrm{e}^{-\gamma \lambda_{ \pm}(r)}\left[K_{o}^{ \pm}-V_{\text {eff }}^{ \pm}(r)\right]^{1 / 2} .
$$

Note that one needs to specify $\dot{\theta}_{o}$ also to determine Equation (22). In addition, one normally wants to know the trajectory in the real space, that is, the acknowledgment of $r=r(\theta)$. Since one has that $v=\mathrm{d} r / \mathrm{d} t=(\mathrm{d} r / \mathrm{d} \theta) \dot{\theta}$ and Equations (22) and (40), it follows that

$$
\theta(r)=\theta_{o}+\frac{l_{\theta}^{2}}{\sqrt{2 m_{2 \pm}^{o}}} \int_{r_{o}}^{r} \frac{m_{2 \pm}(r) \mathrm{e}^{\gamma \lambda_{ \pm}(r)} \mathrm{d} r}{r^{2} \sqrt{K_{o}^{ \pm}-V_{e f f}^{ \pm}(r)}} .
$$

The half-time period (going from aphelion to perihelion $(+)$, or backward $(-))$ can be deduced from Equation (40) as

$$
T_{1 / 2}^{ \pm}=\frac{1}{\sqrt{2 m_{2 \pm}^{o}}} \int_{r_{1}}^{r_{2}} \frac{m_{2 \pm}(r) \mathrm{e}^{\gamma \lambda_{ \pm}(r)} \mathrm{d} r}{\sqrt{K_{o}^{ \pm}-V_{e f f}^{ \pm}(r)}},
$$

where $r_{1}$ and $r_{2}$ are the two return points resulting from the solution of the following equation

$$
V_{\text {eff }}^{ \pm}\left(r_{i}\right)=K_{o}^{ \pm}, i=1,2 .
$$

On the other hand, the trajectory in the space $(r, p)$ is determine by the Hamiltonian (38), and given the same initial conditions, the initial $p_{o}$ and $H_{o}^{ \pm}$are obtained from Equations (38) and (37). Thus, this trajectory is given by

$$
p= \pm \sqrt{\frac{2 m_{2 \pm}^{2}(r)}{m_{2 \pm}^{o}}} \mathrm{e}^{\gamma \lambda_{ \pm}(r)}\left[H_{o}^{ \pm}-V_{e f f}^{ \pm}(r)\right]^{1 / 2} .
$$

It is clear just by looking the expressions (40) and (44) that the trajectories in the spaces $(r, v)$ and $(r, p)$ must be different due to complicated relation (37) between $v$ and $p$ [19].

\section{Mass-Variable Model and Results}

As a possible application, consider that a comet looses material as a result of the interaction with star wind in the following way (for one cycle of oscillation)

$$
m_{2 \pm}(r)=\left\{\begin{array}{l}
m_{2-}\left(r_{2(i-1)}\right)\left(1-\mathrm{e}^{-\alpha r}\right), v<0 \\
\left.m_{2+}\left(r_{2 i-1}\right)-b\left(1-\mathrm{e}^{-\alpha\left(r-r_{2 i-1}\right.}\right)\right), v>0
\end{array}\right.
$$

where the parameters $b>0$ and $\alpha>0$ can be chosen to math the mass loss rate in the incoming and outgoing cases. The index " $i$ " represent the ith-semi-cycle, being $r_{2(i-1)}$ and $r_{2 i-1}$ the aphelion $\left(r_{a}\right)$ and perihelion $\left(r_{p}\right)$ points ( $r_{o}$ is given by the initial conditions, and one has that $\left.m_{2-}\left(r_{o}\right)=m_{o}\right)$. For this case, the functions $\lambda_{+}(r)$ and $\lambda_{-}(r)$ are given by

$$
\lambda_{+}(r)=\frac{1}{\alpha m_{a}} \ln \left(\mathrm{e}^{\alpha r}-1\right),
$$


and

$$
\lambda_{-}(r)=\frac{-1}{\alpha\left(b-m_{p}\right)}\left[\alpha r+\ln \left(m_{p}-b\left(1-\mathrm{e}^{-\alpha\left(r-r_{p}\right)}\right)\right)\right]
$$

where we have defined $m_{a}=m_{2}\left(r_{a}\right)$ and $m_{p}=m_{2}\left(r_{p}\right)$. Using the Taylor expansion, one gets

$$
\begin{aligned}
& \mathrm{e}^{2 \gamma \lambda_{+}(r)} \\
& =\mathrm{e}^{2 \gamma r / m_{a}}\left[1-\frac{2 \gamma}{\alpha m_{a}} \mathrm{e}^{-\alpha r}+\frac{1}{2} \frac{2 \gamma}{\alpha m_{a}}\left(\frac{2 \gamma}{\alpha m_{a}}-1\right) \mathrm{e}^{-2 \alpha r}+\cdots\right],
\end{aligned}
$$

and

$$
\mathrm{e}^{2 \gamma \lambda_{-}(r)}=\frac{\mathrm{e}^{-\frac{2 \gamma r}{\left(b-m_{p}\right)}}}{\left(m_{p}-b\right) \frac{2 \gamma}{\alpha\left(m_{p}-b\right)}} \times\left[1+\frac{2 \gamma}{\alpha\left(m_{p}-b\right)} \frac{\mathrm{e}^{-\alpha\left(r-r_{p}\right)}}{m_{p}-b}+\frac{1}{2} \frac{2 \gamma}{\alpha\left(m_{p}-b\right)}\left(\frac{2 \gamma}{\alpha\left(m_{p}-b\right)}-1\right) \times \frac{\mathrm{e}^{-2 \alpha\left(r-r_{p}\right)}}{\left(m_{p}-b\right)^{2}}+\cdots\right]
$$

The effective potential for the incoming comet can be written as

$$
V_{e f f}^{+}(r)=\left[-\frac{G m_{1} m_{a}}{r}+\frac{l_{\theta}^{2}}{2 m_{a}} \frac{1}{r^{2}}\right] \mathrm{e}^{2 \gamma r / m_{a}}+W_{1}(\gamma, \alpha, r),
$$

and for the outgoing comet as

$$
\begin{aligned}
V_{e f f}^{-}(r) & =\left[-\frac{G m_{1} m_{a}}{r}+\frac{l_{\theta}^{2}}{2 m_{a}} \frac{1}{r^{2}}\right] \\
& \times \frac{\mathrm{e}^{\frac{2 \gamma r}{\left(m_{p}-b\right)}}}{\left(m_{p}-b\right) \frac{2 \gamma}{\alpha\left(m_{p}-b\right)}}+W_{2}(\gamma, \alpha, r),
\end{aligned}
$$

where $W_{1}$ and $W_{2}$ are given in the Appendix. We will use the data corresponding to the sun mass $\left(1.9891 \times 10^{30} \mathrm{Kg}\right)$ and the Halley comet [27] and [28]

$$
\begin{aligned}
& m_{c} \approx 2.3 \times 10^{14} \mathrm{Kg}, r_{p} \approx 0.6 \mathrm{au}, \\
& r_{a} \approx 35 \mathrm{au}, l_{\theta} \approx 10.83 \times 10^{29} \mathrm{Kg} \cdot \mathrm{m}^{2} / \mathrm{s},
\end{aligned}
$$

with a mass lost of about $\delta m \approx 2.8 \times 10^{11} \mathrm{Kg}$ per cycle of oscillation. Although, the behavior of Halley comet seem to be chaotic [29], but we will neglect this fine detail here. Now, the parameters $\alpha$ and " $b$ " appearing on the mass lost model, Equation (45), are determined by the chosen mass lost of the comet during the approaching to the sun and during the moving away from the sun (we have assumed the same mass lost in each half of the cycle of oscillation of the comet around the sun). Using Equation (50) and Equation (51) in the expression (40), the trajectories can be calculated in the spaces $(r, v)$. Figure 1 shows these trajectories using

$\delta m=2 \times 10^{10} \mathrm{Kg} \quad($ or $\delta m / m=0.0087 \%)$ for $\gamma=0$ and (continuos line), and for $\gamma=-3 \mathrm{Kg} / \mathrm{m}$ (dashed line), starting both cases from the same aphelion distance. As one can see on the minimum, dissipation causes to reduce a little bit the velocity of the comet, and the antidissipation increases the comet velocity, reaching a further away aphelion point. Also, when only mass lost is considered $(\gamma=0)$ the comet returns to aphelion point a little further away from the initial one during the cycle of oscillation. Something related with this effect is the change of period as a function of mass lost $(\gamma=0)$. This can be see on Figure 2, where the period is calculated starting always from the same aphelion point $\left(r_{a}\right)$. Note that with a mass lost of the order $2.8 \times 10^{11} \mathrm{Kg}$ (Halley comet), which correspond to $\delta m / m=12 \%$, the comet is well within 75 years period. The variation of the ratio of the change of aphelion distance as a function of mass lost $(\gamma=0)$ is shown on Figure 3. On Figure 4, the mass lost rate is kept fixed to $\delta m / m=0.0087 \%$, and the variation of the period of the comet is calculated as a function of the dissipative-antidissipative parameter $\gamma<0$ (using $|\gamma|$ for convenience). As one can see, antidissipation always wins to dissipation, bringing about the increasing of the period as a function of this parameter. The reason seems to be that the antidissipation acts on the comet when this ones is lighter than when dissipation was acting (dissipation acts when the comet approaches to the sun, meanwhile antidissipation acts when the comet goes away from the sun). Since the period of Halley comets has not changed much during many turns, we can assume that the parameter $\gamma$ must vary in the interval $(-0.01,0] \mathrm{Kg} / \mathrm{m}$. Finally, Figure 5

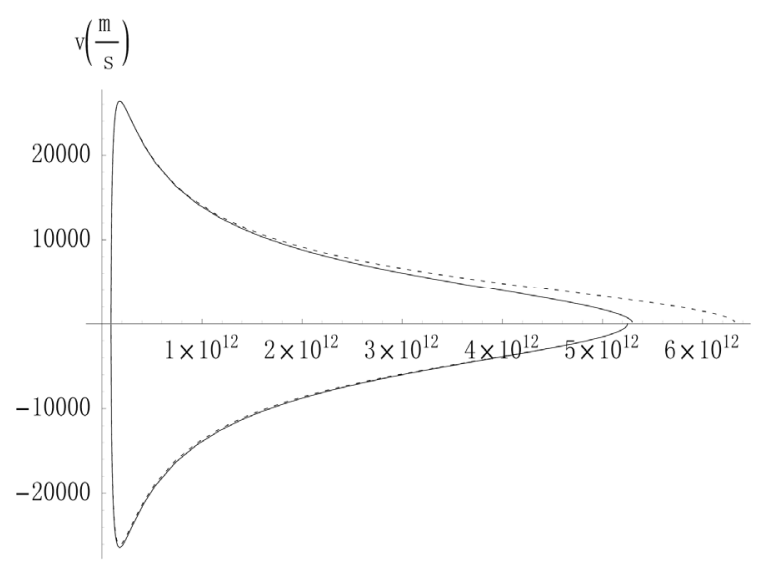

Figure 1. Trajectories in the $(r, v)$ space with $\delta m / m=0.009$. 


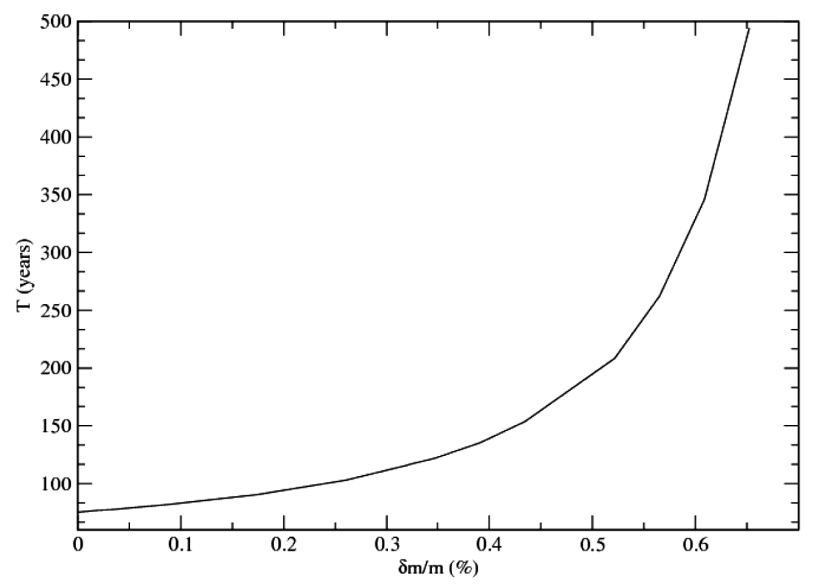

Figure 2. Period of the comet as a function of the mass lost ratio.

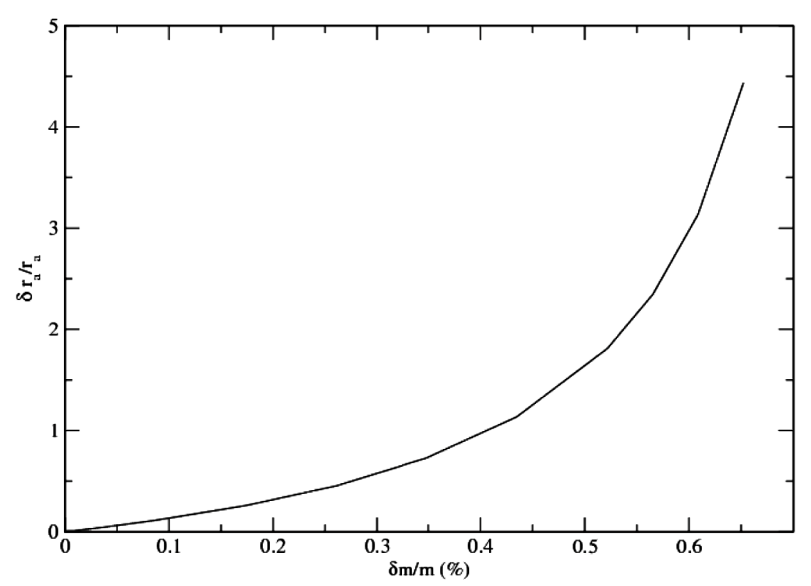

Figure 3. Ratio of aphelion distance change as a function of the mass lost rate.

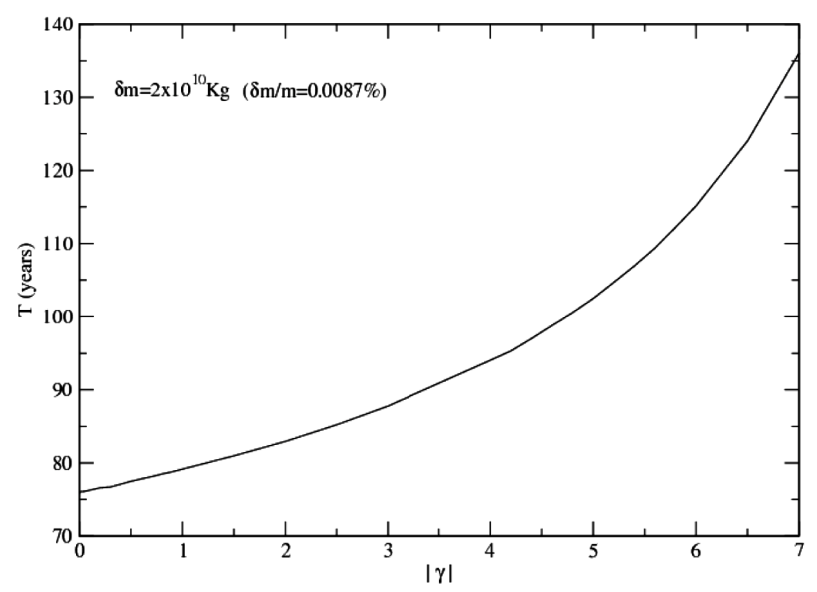

Figure 4. Period of the comet as a function of the parameter $\gamma$.

shows the variation, during a cycle of oscillation, of the ratio of the new aphelion $\left(r_{a}^{\prime}\right)$ to old aphelion $\left(r_{a}\right)$ as a function of the parameter $\gamma$.

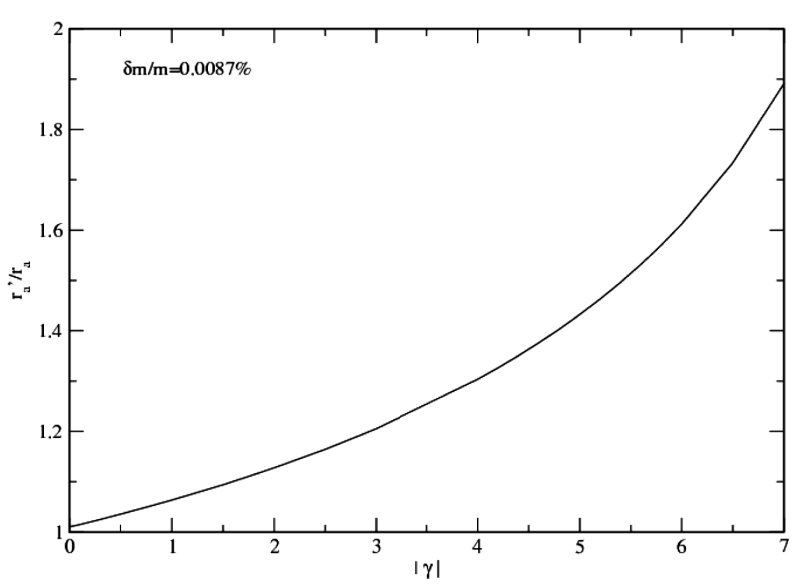

Figure 5. Ratio of the aphelion increasing as a function of the parameter $\gamma$.

\section{Conclusion and Comments}

We have shown that the proposed modified Newton equation for mass variation systems has some problems. Therefore, we have considered that it is better to keep Newton's equations of motion for mass variable systems to have a consistent approach to these problems. Having this in mind, the Lagrangian, Hamiltonian and a constant of motion of the gravitational attraction of two bodies were given when one of the bodies has variable mass and the dissipative antidissipative effect of the solar wind is considered. By choosing the reference system in the massive body, the system of equations is to reduce 1-D problem. Then, the constant of motion, Lagrangian and Hamiltonian were obtained consistently. A model for comet-mass-variation was given, and with this model, a study was made of the variation of the period of one cycle of oscillation of the comet when there are mass variation and dissipation-antidissipation. When mass variation is only considered, the comet trajectory is moving away from the sun, the mass lost is reduced as the comet is farther away (according to our model), and the period of oscillations becomes bigger. When dissipation antidissipation is added, this former effect becomes higher as the parameter $\gamma$ becomes higher.

\section{REFERENCES}

[1] G. López, L. A. Barrera, Y. Garibo, H. Hernández and J. C. Salazar, International Journal of Theoretical Physics, Vol. 43, 2004, p. 1.

[2] H. Gylden, Astronomische Nachrichten, Vol. 109, 1884, pp. 1-6. http://dx.doi.org/10.1002/asna.18841090102

[3] I. V. Meshcherskii, Astronomische Nachrichten, Vol. 132, 1893, p. 93.

[4] I. V. Meshcherskii, Astronomische Nachrichten, Vol. 159, 1902, p. 229. 
[5] E. O. Lovett, Astronomische Nachrichten, Vol. 158, 1902, pp. 337-344. http://dx.doi.org/10.1002/asna.19021582202

[6] J. H. Jeans, MNRAS, Vol. 85, 1924, p. 2.

[7] L. M. Berkovich, Celestial Mechanics, Vol. 24, 1981, pp. 407-429. http://dx.doi.org/10.1007/BF01230399

[8] A. A. Bekov, Astron. Zh., Vol. 66, 1989, p. 135.

[9] C. Prieto and J. A. Docobo, Astronomy \& Astrophysics, Vol. 318, 1997, p. 657.

[10] A. Sommerfeld, "Lectures on Theoretical Physics," Vol. 1, Academic Press, 1964.

[11] A. G. Zagorodny, P. P. J. M. Schram and S. A. Trigger, Physical Review Letters, Vol. 84, 2000, pp. 3594-3597. http://dx.doi.org/10.1103/PhysRevLett.84.3594

[12] O. T. Serimaa, J. Javanainen and S. Varró, Physical Review A, Vol. 33, 1986, pp. 2913-2927. http://dx.doi.org/10.1103/PhysRevA.33.2913

[13] H. A. Bethe, Physical Review Letters, Vol. 56, 1986, pp. 1305-1308. http://dx.doi.org/10.1103/PhysRevLett.56.1305

[14] E. D. Commins and P. H. Bucksbaum, "Weak Interactions of Leptons and Quarks," Cambridge University Press, Cambridge, 1983.

[15] F. W. Helhl, C. Kiefer and R. J. K. Metzler, "Black Holes: Theory and Observation,” Springer-Verlag, 1998. http://dx.doi.org/10.1007/b13593

[16] P. W. Daly, Astronomy \& Astrophysics, Vol. 226, 1989, p. 318.

[17] H. Goldstein, "Classical Mechanics," Addison-Wesley,
1950.

[18] A. R. Plastino and J. C. Muzzio, Celestial Mechanics and Dynamical Astronomy, Vol. 53, 1992, pp. 227-232. http://dx.doi.org/10.1007/BF00052611

[19] G. López, International Journal of Theoretical Physics, Vol. 46, 2007, pp. 806-816. http://dx.doi.org/10.1007/s10773-006-9085-4

[20] C. Møller, "Theory of Relativity," Oxford University Press, Oxford, 1952.

[21] M. Spivak, "Physics for Mathematicians, Mechanics I," Publish or Perish Inc., 2010.

[22] G. López, "Partial Differential Equations of First Order and Their Applications to Physics," World Scientific, 1999. http://dx.doi.org/10.1142/4006

[23] F. John, "Partial Differential Equations," Springer-Verlag, New York, 1974.

[24] J. A. Kobussen, Acta Phys. Austr., Vol. 51, 1979, p. 193.

[25] C. Leubner, Physics Letters A, Vol. 86, 1981, pp. 68-70. http://dx.doi.org/10.1016/0375-9601(81)90166-3

[26] G. López, Annals of Physics, Vol. 251, 1996, pp. 372-383. http://dx.doi.org/10.1006/aphy.1996.0118

[27] G. Cevolani, G. Bortolotti and A. Hajduk, Il Nuovo Cimento C, Vol. 10, 1987, pp. 587-591. http://dx.doi.org/10.1007/BF02507255

[28] J. L. Brandy, Journal of the British Astronomical Association, Vol. 92, 1982, p. 209.

[29] B. V. Chirikov and V. V. Vecheslavov, Astronomy \& Astrophysics, Vol. 221, 1989, p. 146. 


\section{Appendix}

Expression for $W_{1}$ and $W_{2}$ :

$$
\begin{aligned}
W_{1} & =\frac{G m_{2-}^{2}}{m_{2+}^{o}}\left\{-\frac{p(p-1) \mathrm{e}^{(-4+p) \alpha r}}{2 r}+\alpha p E_{i}(\alpha p r)-2 \alpha p(p-1) E_{i}((-4+p) \alpha r)\right. \\
& +\frac{\alpha p^{2}(p-1)}{2} E_{i}((-4+p) \alpha r)+\frac{p(p-1)}{r}\left[\mathrm{e}^{(p-3) \alpha r}+3 \alpha(1-p) r E_{i}((p-3) \alpha r)\right] \\
& \left.+\frac{p(p+3)}{2}\left[-\frac{\mathrm{e}^{(p-2) \alpha r}}{r}+\alpha(p-2) E_{i}((p-2) \alpha r)\right]+\frac{p+2}{r}\left[\mathrm{e}^{(p-2) \alpha r}+\alpha(p-1) r E_{i}((p-1) \alpha r)\right]\right\} \\
& +\frac{l_{\theta}^{2}}{2 m_{2+}^{2} r^{2}}\left\{\frac{p(p-1)}{2} \mathrm{e}^{(p-2) \alpha r}-p \mathrm{e}^{(p-1) \alpha r}-\alpha p(p-1) \mathrm{e}^{(p-2) \alpha r}+\frac{\alpha p(p-1)}{2} \mathrm{e}^{p \alpha r}\right. \\
& +\frac{\alpha^{2} p(p-1) r}{2} \mathrm{e}^{(p-2) \alpha r}+p \alpha r \mathrm{e}^{(p-1) \alpha r}-p^{2} \alpha r \mathrm{e}^{(p-1) \alpha r}-p^{2} \alpha^{2} r^{2} E_{i}(p \alpha r) \\
& -\frac{\alpha^{2}(p-2)^{2} p(p-1) r^{2}}{2} E_{i}((p-2) \alpha r)+p \alpha^{2} r^{2} E_{i}((p-1) \alpha r) \\
& \left.-2 \alpha^{2} p^{2} r^{2} E_{i}((p-1) \alpha r)+p^{3} \alpha^{2} r^{2} E_{i}((p-1) \alpha r)\right\}
\end{aligned}
$$

where $m_{a}$ is the mass of the body at the aphelion, and we have made the definitions

$$
\begin{aligned}
p=\frac{2 \gamma}{\alpha m_{a}} . \\
W_{2}=\frac{G m_{2-}^{2}}{m_{2+}^{o}}\left\{\frac{\mathrm{e}^{(q-2) \alpha r}}{r}\left[1+\frac{q(q-1)}{2\left(m_{p}+\alpha q\right)} \mathrm{e}^{2 q \alpha r}+\frac{2 q}{m_{p}+\alpha q} \mathrm{e}^{q \alpha r}\right]\right. \\
+q \alpha E_{i}(q \alpha r)-\frac{q(q-1) \mathrm{e}^{2 q \alpha r}}{\left(m_{p}+\alpha q\right)^{2} r}\left[\mathrm{e}^{(q-3) \alpha r}-\alpha(q-3) r E_{i}((q-3) \alpha r)\right] \\
+\frac{q \mathrm{e}^{q \alpha r}}{\left(m_{p}+\alpha q\right) r}\left[\mathrm{e}^{(q-3) \alpha r}-\alpha(q-3) r E_{i}((q-3) \alpha r)\right]-2 \alpha E_{i}((q-2) \alpha r) \\
+\alpha q E_{i}((q-2) \alpha r)-\frac{q(q-1) \alpha \mathrm{e}^{2 q \alpha r}}{\left(m_{p}+\alpha q\right)^{2}} E_{i}((q-2) \alpha r)+\frac{q^{2}(q-1) \alpha \mathrm{e}^{2 q \alpha r}}{2\left(m_{p}+\alpha q\right)^{2}} E_{i}((q-2) \alpha r) \\
+\frac{4 \alpha \mathrm{e}^{q \alpha r}}{m_{p}+\alpha q} E_{i}((q-2) \alpha r)+\frac{2 q^{2} \alpha \mathrm{e}^{q \alpha r}}{\left(m_{p}+\alpha q\right) r} E_{i}((q-2) \alpha r) \\
\left.+\frac{2}{r}\left[\mathrm{e}^{(q-1) \alpha r}-(q-1) \alpha r E_{i}((q-1) \alpha r)\right]+\frac{q \mathrm{e}^{q \alpha r}}{\left(m_{p}+\alpha q\right) r}\left[\mathrm{e}^{(q-1) \alpha r}-(q-1) \alpha r E_{i}((q-1) \alpha r)\right]\right\} \\
+\frac{l_{\theta}^{2}}{2 m_{2+}^{2}\left(m_{p}+\alpha q\right)^{q}}\left\{-\frac{q \alpha \mathrm{e}^{q \alpha r}}{r}+q^{2} \alpha^{2} E_{i}(q \alpha r)+\frac{q(q-1) \mathrm{e}^{(3 q-2) \alpha r}}{2\left(m_{p}+\alpha q\right)^{2} r^{2}}\right. \\
\left.\quad-\frac{q \mathrm{e}^{(2 q-1) \alpha r}}{\left(m_{p}+\alpha q\right) r^{2}}\left[-1+\alpha r+q \alpha r+(q-1)^{2} \alpha^{2} r^{2} \mathrm{e}^{(1-q) \alpha r} E_{i}((q-1) \alpha r)\right]\right\}
\end{aligned}
$$


where $m_{p}$ is the mass of the body at the perihelion, and we have made the definition

$$
q=\frac{2 \gamma}{\alpha\left(m_{p}-b\right)}
$$

and the function $E_{i}$ is the exponential integral,

$$
E_{i}(z)=\int_{-z}^{\infty} \frac{\mathrm{e}^{-t}}{t} \mathrm{~d} t
$$

